\title{
HUMANIZAÇÃO DO PARTO NA PERSPECTIVA DA EQUIPE DE ENFERMAGEM DE UM CENTRO DE PARTO NORMAL
}

José Wellington Silva de Moura John Carlos de Souza Leite ${ }^{1}$ Vinicius Rodrigues de Oliveira ${ }^{1}$ João Paulo Xavier Silva ${ }^{1}$

\author{
https://orcid.org/0000-0002-5459-3310 \\ https://orcid.org/0000-0002-0183-6913 \\ https://orcid.org/ 0000-0002-9915-0062 \\ https://orcid.org/ 0000-0003-3082-9373
}

Objetivo: Compreender a percepção de uma equipe de enfermagem de um Centro de Parto Normal acerca da assistência ao par to humanizado. Método: Trata-se de uma pesquisa descritiva, qualitativa, realizada com profissionais de enfermagem de um Cen tro de Parto Normal, em um município do interior do Ceará. Os dados foram analisados segundo a análise categorial temática, utilizando-se o software IRAMUTEQ para processamento dos dados. Resultados: Os participantes do estudo reconhecem a relevância de seu trabalho e identificam a classe da enfermagem como protagonista na assistência humanizada. Esses apresentam a percepção de parto humanizado relacionado à autonomia da mulher, além disso, entendem que o processo de humanização se inicia desde a entrada da mulher no centro de parto. Conclusão: Os profissionais de enfermagem demonstraram conhecimento científico sobre a assistência ao parto humanizado e sobre as práticas de humanização para a parturiente.

Descritores: Parto humanizado; Cuidados de enfermagem; Profissionais de enfermagem; Enfermagem obstétrica.

\section{BIRTH HUMANIZATION IN THE PERSPECTIVE OF THE NURSING TEAM OF A NATURAL BIRTH CENTER}

Objective: Comprehend the perspective of a nursing team in a natural birth center about the assistance on humanized childbirth Method: this is a descriptive, qualitative research carried out with nursing professionals of a natural birth center, in a municipality in the interior of Ceará. The data were analyzed according to the thematic categorical analysis, using IRAMUTEQ software in data processing. Results: The study participants recognize the relevance of their work and identify the nursing class as the protagonist in humanized care. This present the perception of humanized birth related to women's autonomy, also, they understand that the humanization process begins when the woman enters the birth center. Conclusion: Nursing professionals demonstrated scientific knowledge about humanized childbirth assistence, and about humanization practices for parturient women.

Descriptors: Humanizing Delivery; Nursing Care; Nurse Practitioners; Obstetric Nursing.

\section{HUMANIZACIÓN DEL PARTO NA PERSPECTIVA DEL EQUIPO DE ENFERMERÍA DE UN CENTRO DE PARTO NORMAL}

Objetivo: Comprender la percepción de un equipo de enfermería en un Centro de Parto Normal acerca de la asistencia humanizada para el parto. Metodo: Esta es una investigación descriptiva y cualitativa, realizada con profesionales de enfermería de un Centro de Parto Normal, en una ciudad del interior de Ceará. Los datos se analizaron de acuerdo con el análisis categórico temático, utilizando un software IRAMUTEQ en el procesamiento de datos. Resultados: Los participantes del estudio reconocen la relevancia de su trabajo e identifican a la clase de enfermería como protagonista en la atención humanizada. Estos presentan la percepción del parto humanizado en relación con la autonomía de la mujer, además, entienden que el proceso de humanización comienza desde el ingreso de la mujer al centro de parto. Conclusión: Los profesionales de enfermería demostraron conocimiento científico sobre el cuidado del parto humanizado y sobre las prácticas de humanización para mujeres parturientas.

Descriptores: Parto Humanizado; Atención de Enfermería; Enfermeras Practicantes; Enfermería Obstétrica.

1Manuscrito extraido da monografia intitulada: "Percepção dos profissionais de enfermagem acerca da assistência ao parto humanizado", apresentada em 2019, para o Curso de Graduação em Enfermagem da Universidade Regional do Cariri. ${ }^{2}$ Universidade Regional do Cariri (URCA) - Campus Iguatu, CE,Brasil.

Autor Correspondente: Vinicius Rodrigues de Oliveira Email: viniciusrodriguesvro@gmail.com Recebido: 02/3/20 Aceito: 04/6/20 


\section{INTRODUÇÃO}

O parto humanizado consiste em um conjunto de práticas e procedimentos que visam um processo de parto de forma acolhedora e mais humana, menos medicalizado e que utilize menos intervenções desnecessárias, como o uso rotineiro de amniotomia precoce (rompimento da bolsa) e ocitocina para induzir o trabalho de parto. Dessa forma são utilizadas práticas e métodos naturais que tornam o parto mais humanizado, tais como: massagens, técnicas de respiração, banhos, além do apoio psicológico que deve ser ofertado para parturiente e sua familial.

Humanizar o parto não consiste somente em realizar ou não procedimentos e práticas, mas sim permitir que a mulher se torne a personagem principal nesse cenário, como forma de evitar que ela seja apenas uma espectadora desse momento, dando-lhe direito de escolha nos processos decisórios na assistência humanizada²

A assistência humanizada deve acontecer bem antes da realização do parto, tendo início no começo da gravidez, nas primeiras consultas de pré-natal, dando continuidade no parto e pós-parto ${ }^{3}$. Essa assistência vem sendo implementada no Centro de Parto Normal (CPN) que é regulamentado pela Portaria no 11, de 7 de janeiro de 2015, instituída pela Ministério da Saúde (MS) no âmbito do Sistema Único de Saúde (SUS), estabelecendo assim a implantação e capacitação CPN para a assistência materna e ao Recém-Nascido (RN) na hora do parto e ao nascer ${ }^{4}$

Essa alternativa para o parto foge do modelo assistencial mecanicista, medicalizador e intervencionista do parto hospitalar, visando uma assistência individualizada, de forma integral e humanizada, sem a utilização de intervenções desnecessárias e respeitando a fisiologia do parto e da mulher ${ }^{1}$.

Nesse contexto, se faz necessária a presença da equipe de saúde, destacando-se atuação da equipe de Enfermagem na assistência ao parto humanizado, com intuito de oferecer acolhimento de qualidade, apoio, suporte afetivo, psicológico, físico e emocional para a parturiente e a família, como também estimular a participação ativa desta e seu acompanhante, utilizar as práticas humanizadas, priorizar o protagonista da mulher no parto e respeitar a presença do companheiro ou de outros acompanhantes no momento do parto ${ }^{5-6}$.

Contudo, para que a assistência humanizada ao parto seja realizada de forma qualificada e segura é importante que os profissionais de Enfermagem possuam uma formação fundamentada nos princípios humanistas, que tenham conhecimento necessário e estejam qualificados para atuar nessa assistência ${ }^{2}$.

Assim essa pesquisa teve como objetivo compreender a percepção dos profissionais de uma equipe de Enfermagem de um CPN acerca da assistência ao parto humanizado.

\section{MÉTODO}

\section{Tipo de estudo}

Trata-se de uma pesquisa exploratória, descritiva, sob a abordagem qualitativa.

\section{Participantes do estudo}

Os participantes do estudo foram definidos por meio da amostragem não probabilística intencional'. A equipe de Enfermagem do CPN investigada neste estudo conta com 13 profissionais, sendo sete técnicos de enfermagem e seis enfermeiros, entretanto a pesquisa foi realizada com uma amostra de 11 profissionais de enfermagem, devido duas recusas em participar do estudo. O critério de inclusão para participação foi: os profissionais de Enfermagem que possuíam uma vivência de no mínimo seis meses no CPN, a escolha desse período de tempo justifica-se pelo fato de que com seis meses o indivíduo já tem adquirido experiência profissional, além disso é essencial para se situarem com a realidade na qual atuam. Os critérios de exclusão foram: os profissionais que estavam em licença maternidade, os que estiveram afastados por atestado médico e os profissionais que não compareceram ao local no período de coleta.

\section{Local de estudo}

O estudo foi realizado em um CPN, de um município do interior do Ceará, vinculado ao hospital regional do município e fornece assistência não apenas para o municipio sede, mas também a outros nove municípios circunvizinhos, com base na regionalização sanitária.

\section{Coleta de dados}

Os dados foram coletados no período de abril e maio de 2019, por meio de uma entrevista semiestruturada. Antes de iniciar a coleta, foi realizado um teste piloto do instrumento utilizado, com a participação de profissionais de Enfermagem atuantes no CPN de um município cearense cuja realidade se assemelha a da cidade onde o estudo foi desenvolvido. Após a realização do teste, não houve a necessidade de ajuste no instrumento de coleta. As entrevistas não puderam ser agendadas previamente, pois o pesquisador não conseguiu contato dos participantes ou escala de plantões, assim, foi diariamente ao CPN até conseguir finalizar a coleta. Inicialmente a comunicação entre profissional e pesquisador era feita por intermédio da recepcionista do CPN que verificava a disponibilidade do profissional no dia, tendo disponibilidade e interesse em participar da pesquisa os dados eram coletados. A entrevista foi realizada no ambiente de trabalho dos participantes em um espaço sigiloso e tranquilo, registrada em áudio por meio de equipamento eletrônico (smartphone) e abordou as características sociodemográficas e profissionais da equipe de Enfermagem, o conhecimento quanto à assistência ao parto humanizado, as práticas humanizadas realizadas pelos 
profissionais de Enfermagem durante o parto e a assistência de enfermagem ao parto humanizado.

Procedimentos de análise e tratamento de dados

As informações obtidas foram apresentadas na forma descritiva e analisados segundo a análise temática, através de três etapas: 1a pré-análise: leitura do material e formatação do corpus textual; 2 a exploração do material: processamento de dados por meio do software Interface de R pour les Analyses Multidimensionnelles de Textes et de Questionnaires (IRAMUTEQ); e 3a tratamento dos resultados: elaboração de categorias temáticas, seguinte a discussão embasada mediante a literatura conveniente ao assunto ${ }^{8}$. No processamento de dados adotou-se a Classificação Hierárquica Descendente (CHD) que resultou em quatro classes distintas. Cada classe resultante é formada pelas palavras mais expressivas, ou seja, as palavras com a maior frequência. A classe 4, denominada "Assistência de enfermagem ao parto humanizado", representa, 31,7\% dos segmentos de textos, a classe 3, denominada "Práticas Humanizadas no Parto" representa, 25,8\% dos segmentos de texto, a classe 2 , denominada "Parto Humanizado" representa, 20\% dos segmentos de texto, a classe 1, denominada "Importância da Enfermagem ao Parto Humanizado" de representa, 22,5\% dos segmentos de texto.

\section{Procedimentos éticos}

A pesquisa se desenvolveu de acordo com as determinações estabelecidas pelas Resoluções № 466/12 e № 510/2016 do Conselho Nacional de Saúde (CNS) que dispõem acerca dos preceitos éticos e legais para pesquisa com seres humanos. $O$ projeto foi submetido ao Comitê de Ética em Pesquisa (CEP) da Universidade Regional do Cariri (URCA) sendo aprovado sob Parecer $\mathrm{N}^{\circ}$ 3.183.202. Todos os integrantes da pesquisa foram devidamente informados e esclarecidos das finalidades da pesquisa e a importância da participação deste para o estudo por meio do Termo de Consentimento Livre e Esclarecido. Foram utilizados pseudônimos (P1, P2, P3, sucessivamente), para preservar a identidade dos participantes.

\section{RESULTADOS}

Dentre os participantes do estudo nove são do sexo feminino e dois masculino, em relação faixa etária esta variou de 18 a 41 anos. No que diz respeito a profissão, a equipe é formada por seis técnicos em enfermagem e cinco enfermeiros, uma característica importante da formação dos Enfermeiros é a especialização na área de Enfermagem Obstétrica. No que se refere o tempo de atuação no CPN, variou entre seis meses a dois anos, 10 dos profissionais já atuavam no serviço entre um ano a dois anos. Em relação a capacitações dos profissionais para assistência ao parto humanizado, oito afirmaram não terem participado de nenhuma capacitação, e apenas três relataram a participação em alguma capacitação. As capacitações vivenciadas pelos sujeitos do estudo não foram ofertadas pela instituição ao qual trabalham.

Quanto ao processamento das informações no software IRAMUTEQ, o conteúdo analisado foi categorizado em quatro classes, dispostas a seguir.

Figura 1 - Dendrograma da CHD: Percepção dos profissionais de enfermagem acerca da assistência ao parto humanizado. Ceará, Brasil, 2020.

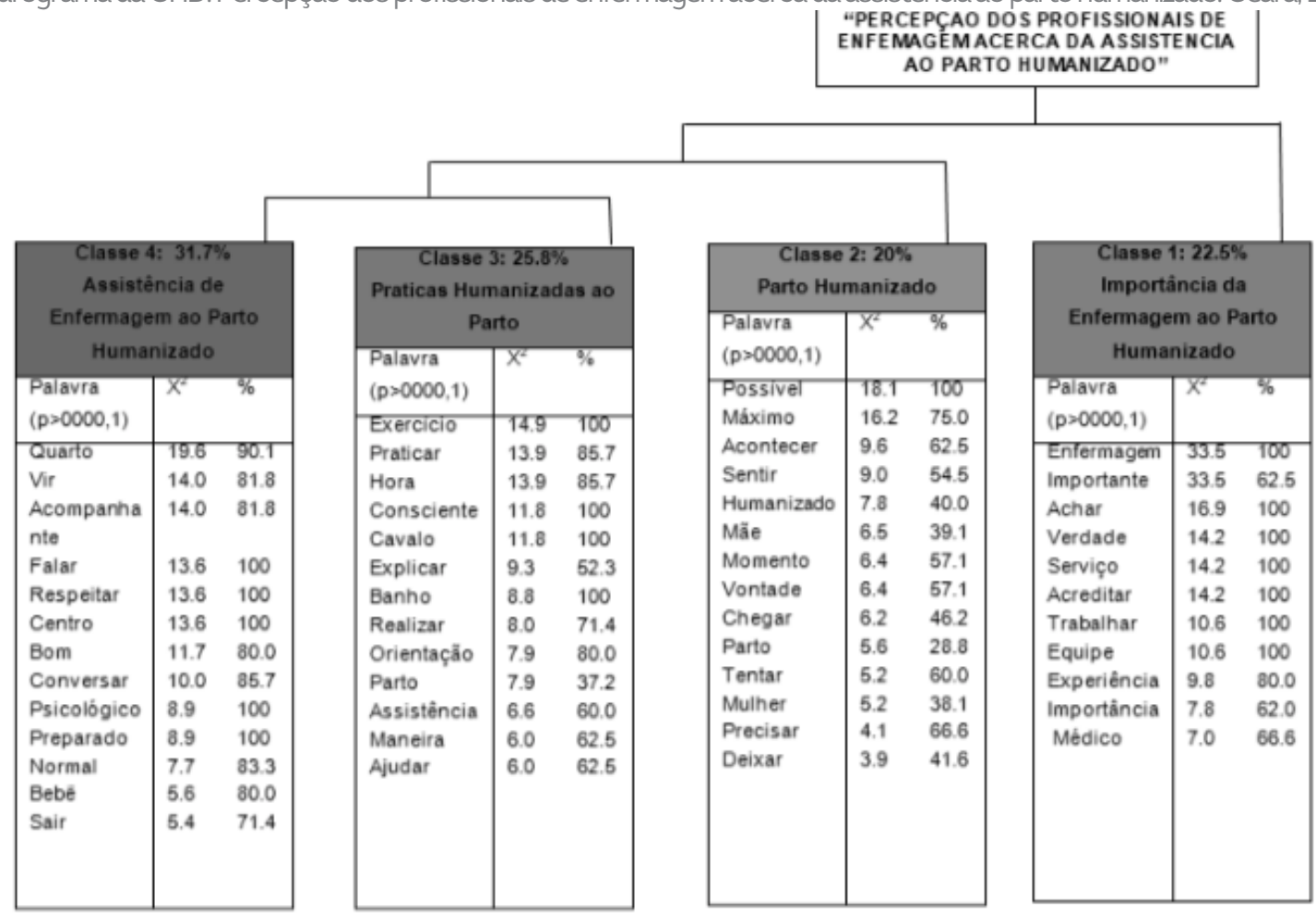




\section{Classe 1: Importância da Enfermagem ao Parto Humanizado}

Essa classe faz menção a importância da Enfermagem no parto humanizado. O estudo demonstra a relevância do trabalho de enfermagem e identifica essa classe como protagonistas na assistência humanizada.

[...] Enfermagem atua como enfermeiro, psicólogo, assistente social, a ta ali pra tranquilizar a paciente pra passar pra ela a nossa experiência para conduzir bem esse trabalho de parto ser bem conduzido, que às vezes nem o médico faz tão bem esse serviço, mas a gente tem esse olhar, apurado com essa paciente, de explicar de ter um certo cuidado com a paciente pra conduzir bem esse trabalho de parto (P2).

[...] a enfermagem é considerada como a profissão que pode dizer que mais humanizada, e como a gente passa por vários cursos e na faculdade a gente tem cadeiras que orientam e muito bem e o médico ele é mais mecânico (Pll).

[...] eu acho de muita importância, nosso trabalho é fundamental, não que dos outros seja até dos serviços gerais, eu acho que todo trabalho em conjunto ele é muito, é importante, o nosso é porque a gente acompanha do começo ao fim, da admissão da paciente a todo processo de trabalho de parto, a hora do parto, pós-parto até a alta (P7).

\section{Classe 2: Parto Humanizado}

Essa classe se refere ao conhecimento dos profissionais em relação ao parto humanizado. Observou-se que a grande maioria dos participantes apresentam a percepção de parto humanizado relacionado à autonomia da mulher e respeito as suas escolhas.

Parto humanizado ele é aquele ato onde o profissional busca desmedicalizar o parto o máximo possivel e com isso deixando a mulher tomando o controle do trabalho de parto bem como procurar sempre oferecer algumas alternativas para que elas se sinta mais à vontade (P4)
O parto humanizado pra mim é um parto em que você dê condições a mãe de ter uma segurança sobre a vida que ela vai trazer ao mundo dar suporte $e$ explicar como vai acontecer (Pll).

[...] como você deve acolher de forma que ela se sinta mais à vontade possivel em relação a posições que a mulher prefira muitas vezes a tem a posição horizontal sendo parto humanizado, mas se a mulher prefere aquela posição então ela é autora do seu próprio momento (P5).

O parto humanizado é aquele parto que a mulher ela é assistenciada do começo ao fim, e que ela tem um acompanhante da escolha dela [...] a gente conversa com ela, explica pra ela, pergunta pra ela se ela realmente está preparada, se ela tem alguma dúvida (P6).

[...] ela sentir o momento do parto é um momento muito especial para ela e ela sentir à vontade o máximo possivel nesse parto e a fazer algumas manobras de conforto deixar a paciente a vontade (P2)

\section{Classe 3: Práticas Humanizadas no Parto}

O principal aspecto dessa classe diz respeito às práticas humanizadas realizadas ao parto. Através do processamento e análise de dados verificou-se que os participantes entendem as práticas como uso do cavalinho, exercício com a bola, banho, diálogo, orientações para o parceiro, deambulação e massagem de alívio, estão relacionadas e fazem parte de uma assistência humanizada.

[...] a gente começa a dar as orientações a ela, primeiramente a gente coloca ela na bola, faz os exercícios, explica pra ela a importância desses exercícios pra ajudar ela no parto (P6)

[...] ensinar a mulher os exercicios da bola, do cavalo que ela pode ficar de cócoras, agachamento, ajudar o pai nesse processo que as vezes o pai quer participar do parto, mas eles não sabem participar (PI).

Enferm. Foco 2020; 11 (3): 202-209 
[...] quando está na bola na posição que ela faz vai aliviar a dor quando vem a contração e são práticas que a gente realiza exercícios, massagens, banho, banho alivia muito a dor da mãe (Pll).

\section{[...] diretamente com a paciente} mesmo orientações, orientações de deambular, de fazer a bola, de fazer até o cavalinho, o agachamento, explicar a ela as maneiras que podem abreviar esse parto de tornar esse parto menos doloroso pra ela (P2).

\section{Classe 4: Assistência de enfermagem ao parto humanizado}

As palavras que melhor se relacionam a esta classe fazem menção a como a assistência de enfermagem ao parto humanizado é iniciada, desde a entrada da mulher no centro de parto, até a assistência em si.

[...] a mãezinha chega a gente ajuda, orienta o que ela pode fazer pra poder a ajudar o parto, dar confiança, a gente chega no quarto a mãezinha tá cheia de dor, a gente conversa um pouco com ela tranquiliza mais, e passa confiança pra ela (P3).

Vai desde do atendimento da mulher, da gestante, a forma de você atender a gestante na triagem de forma humanizada, respeitando as dores, geralmente quando elas vem pra cá vem com muita contração, como aqui é centro de parto elas vão vim para parir, e tem que respeitar o tempo de contração da paciente $(\mathrm{Pl})$.

A gente acolhe a mãe assim que ela chega, no consultório a gente acolhe a mãe conversa explicando os procedimentos que serão realizados [...] (Pll).

[...] Elas chegam no quarto, os quartos são maiores são individuais a mulher tem mais privacidade, respeitar o leito da mulher, respeitar as posições que a mulher quer pra ter o bebe mas sempre tendo o bom senso (P4).
Nesta mesma classe também, torna-se evidente o respeito e valorização que os profissionais de enfermagem têm em relação a presença do acompanhante.

Pra gente se colocar no lugar do outro, deixar a paciente a vontade, respeitar a vontade da paciente de conter o acompanhante que ela quiser no quarto, o esposo ou de preferência da paciente (PlO).

[...] no parto, mesmo no quarto respeitar a escolha do acompanhante dela aqui no centro de parto acho que é único setor que respeita a escolha do acompanhante, até por ter mais estrutura (P2).

[...] aquela pessoa que ela considera o ícone da família dela o marido por exemplo pai e mãe e qualquer um outro parente ou acompanhante deposite confiança naquela pessoa então ter a confiança do profissional e mais a confiança do acompanhante isso se torna muito bom (P9)

\section{DISCUSSÃO}

Os participantes do estudo referem que equipe de Enfermagem é imprescindivel em todo processo do parto, visto que a sua atuação se faz necessária para gerar segurança e liberdade a parturiente, fazendo com que a mesma se sinta mais à vontade possivel e bem acolhida pela equipe? . Por meio, da assistência provida pelos profissionais de Enfermagem é possivel proporcionar a mulher o primeiro contato pele a pele da com o bebê, como também as primeiras orientações sobre os cuidados em geral do bebêe $\hat{e}^{10}$.

No parto humanizado, os profissionais de Enfermagem atuam com vistas à garantir a parturiente uma maior segurança e conforto, sempre de forma respeitosa e com diálogo. Destarte, cria-se um vinculo com a paciente, o que possibilita o profissional reconhecer as fragilidades e assim compreender quais as intervenções devem ser executadas ${ }^{5}$.11.

A atuação da Enfermagem frente ao parto humanizado requer de um profissional qualificado, sem prejulgamentos, sem danos e sem intervenções desnecessárias, além da disponibilidade de meios tecnológicos e infraestrutura apropriada das instituições. Aspectos como respeito, comunicação ativa, auxilio e orientação denotam o quão é importanteéa assistência humanizada do profissional de enfermagem diante do parto ${ }^{12}$.

A Enfermagem no cenário obstétrico é fundamental e insubs- 
tituível, sobretudo no parto humanizado. Pois a mesma adota uma assistência empática, com equidade, de forma integral e individualizada para a parturiente. Diminuindo os anseios da mesma no processo de parto, proporcionando-a coragem e segurança ${ }^{13-14}$.

Diante disso, é indispensável que a equipe de enfermagem esteja sensibilizada e capacitada para exercer essas funções de forma mais qualificada possivel, portanto se faz importante que os programas de educação continuada das instituições de saúde abordem essa temática com maior frequência ${ }^{15}$.

Alguns autores destacam pontos importantes sobre o parto humanizado, aspectos como o cuidado integral, o respeito a fisiologia do parto, a desmedicalização do processo de parto, o ato de ouvir e respeitar escolha do acompanhante, além da utilização de práticas de humanização do parto que diminuem o desconforto mental e físico e da redução de intervenções desnecessárias que prejudicam o parto são recorrentes na literatura, 5,16-17

Fortalecendo esses aspectos, a Organização Mundial da Saúde (OMS) disponibilizou uma publicação intitulada Intrapartum care for a positive childbirth experience, contendo recomendações e não recomendações que devem ser seguidas para uma assistência segura, positiva e humanizada ${ }^{18}$. É imprescindivel que os profissionais de enfermagem saibam o significado da humanização no parto.

Nesta pesquisa os profissionais de Enfermagem entendem a promoção de medidas humanizadas no processo de parto são de suma importância para que esse momento seja vivenciado de uma forma confiante e enriquecedora para a mulher. Para que isso aconteça é necessário que o profissional, trate a mulher de forma humana, acolha, escute-a, respeite as posições, o tempo de contração da mulher, explique-a os procedimentos que serão realizados no parto. Além disso, é importante evitar medidas intervencionistas desnecessárias, e respeitar privacidade e a autonomia da parturiente $e^{19}$.

Corroborando com os achados desse estudo, observa-se que assistência desumanizada no processo de parto pode ocasionar uma experiência traumática e negativa para parturiente. Isto está relacionado com a falta de respeito do profissional com a autonomia da cliente, a impaciência durante o trabalho de parto, as dificuldades e limitações que a usuária apresenta ${ }^{20}$.

Nesse contexto, é fundamental a presença de uma equipe humanizada, pois através de uma assistência qualificada pode-se dar mais segurança e confiança a mulher e sua família, tornando-os mais relaxados e calmos, diminuindo, assim, problemas interpessoais, emocionais e físicos ${ }^{21-22}$

Para os participantes, o respeito da presença e escolha do acompanhante do paciente também é um dos pontos que reforça a assistência humanizada. A presença do acompanhante na hora do parto, é assegurada pela Lei 11.108 , de abril de $2005^{23}$ e é essencial para resgatar a efetividade, a referência familiar, como também o contexto psicológico e emocional, tão vulneráveis nos ambientes hospitalares ${ }^{24}$.

No que se refere ao direito à acompanhante, pesquisas apontam que as mulheres que possuem um acompanhante no momento do parto sentem-se mais seguras e calmas no decorrer desse processo, visto que a sua presença contribui para um menor tempo de trabalho de parto ${ }^{25-26}$.

\section{LIMITAÇÕES DO ESTUDO}

A limitação desse estudo está relacionada a sua realização em um único $\mathrm{CPN}$, como também a dificuldade na coleta de dados devido a sobrecarga de trabalho no contexto dos profissionais da equipe de enfermagem. Desse modo, sugere-se a realização de novos estudos, com mais participantes e em múltiplos centros de parto, que possam vir a contemplar aspectos ainda não elucidados nessa pesquisa.

\section{Contribuições para a prática}

O estudo contribui para reflexão dos profissionais de enfermagem frente a assistência que é desenvolvida ao parto humanizado e situa esses trabalhadores em relação aos impactos positivos que a assistência humanizada e segura produz no serviço e, sobretudo na saúde da parturiente. Ademais, o estudo promove o reconhecimento e valorização da equipe de enfermagem na adoção de práticas humanizadas de parturição.

\section{CONSIDERAÇÕES FINAIS}

Observou-se a importância da assistência do profissional de enfermagem ao parto humanizado, visto que os cuidados realizados pelos profissionais de enfermagem no processo de parto são essenciais para que esse momento seja benéfico para as parturientes, e que as mesmas se sintam confortáveis e seguras.

Em relação ao conhecimento, constatou-se que os profissionais de enfermagem demonstraram conhecimento científico satisfatório sobre a assistência ao parto humanizado e sobre as práticas de humanização para a parturiente no processo de parto, como também identificaram que consequências podem ser apresentadas mediante uma assistência não humanizada.

Notou-se que muitas práticas consideradas importantes para o parto humanizado são colocadas em ação. Contudo, a realidade ainda apresenta desafios a serem superados, dentre eles a fragilidade na educação continuada da equipe de enfermagem.

Contribuições dos Autores: José Wellington Silva de Moura: Concepção e desenho do estudo; coleta, análise e interpretação dos dados. John Carlos de Souza Leite: Concepção e desenho do estudo; revisão crítica do manuscrito. Vinícius Rodrigues de Oliveira: Redação e revisão crítica do manuscrito. João Paulo Xavier Silva: Revisão crítica do manuscrito; aprovação da versão final a ser publicada. 


\section{REFERÊNCIAS}

1. Barros LP, Souza CLT, Gonçalves LF, Gonzaga LN, Paula TA, Silva AM. O parto humanizado e seu impacto na assistência à saúde. RESU [Internet]. 2015 [cited 2018 Set 20]; 3(2):64-71. Available from: http://periodicos.unievangelica.edu.br/ index.php/educacaoemsaude/article/view/1387/1271.

2. Nascimento FCV, Silva MP, Viana MRP. Assistência de enfermagem no parto humanizado. Rev Pre Infec e Saúde [Internet]. 2018 [cited 2018 Set 20]; 4:6887. Available from: https://doi.org/10.26694/repis.v4i0.6887.

3. Silva DC, Rodrigues ARG, Pimenta CJL, Leite ES. Perspectiva das puérperas sobre a assistência de enfermagem humanizada no parto normal. REBES [Internet]. 2015 [cited 2018 Set 20]; 5(2):50-56. Available from: https://www.gvaa. com.br/revista/index.php/REBES/article/view/3660/3317.

4. Ministério da Saúde (BR). Portaria no 1l, de 7 de janeiro de 2015. Redefine as diretrizes para implantação e habilitação de Centro de Parto Normal (CPN), no âmbito do Sistema Único de Saúde. Diário Oficial República Federativa do Brasil, 7 de jan de 2015; Seção 1. [Internet]. 2015 [cited 2018 Set 20]. Available from: https://bvsms.saude.gov.br/bvs/saudelegis/ gm/2015/prt0011_07_01_2015.html

5. Almeida OSC, Gama ER, Bahiana PM. Humanização do parto: a atuação dos enfermeiros. Revista Enfermagem Contemporânea [Internet]. 2015 [cited em 2018 Set 21]; 4(1):79-90. Available from: https://doi.org/10.17267/2317-3378rec. v4i1.456.

6. Possati ABI, Prates LA, Cremonese L, Scarton J, Alves CN, Ressel LB. Humanization of childbirth: meanings and perceptions of nurses. Esc. Anna Nery Rev. Enferm [Internet]. 2017 [cited 2018 Set 21]; 21(4):1-6. Available from: http:// dx.doi.org/10.1590/2177-9465-ean-2016-0366.

7. Meyer, RD. A atuação do profissional de Gestão da Informação frente à pesquisa de marketing de uma empresa. Curitiba. Monografia [Bacharelado em Gestão da Informação] - Universidade Federal do Paraná; 2004. [Internet]. 2004 [cited 2018 Set 20]. Available from: https://acervodigital.ufpr.br/bitstream/handle/1884/48759/TCC\%20\%20Rute\%20 Daniele\%20Meyer\%20-\%20Monografia.pdf?sequence=18isAllowed=y.

8. Minayo, MC. O desafio do conhecimento: pesquisa qualitativa em saúde. 14ạ edição. São Paulo: Hucitec; 2014

9. Takemoto AY, Corso MR. Parto humanizado e a assistência de enfermagem: uma revisão da literatura. Arquivo de Ciência da Saúde [Internet]. 2015 [cited 2019 Jun 5]; 17(2):117-127. Available from: https://doi.org/10.25110/arqsaude. v17i2.2013.5002

10. Souza R, Alves V, Rodrigues D, Branco M, Lopes F, Santos M. Knowledge of nurses about the clinical management of breastfeeding: knowledge and practices. Rev Fun Care Online. [Internet]. 2019 [cited 2019 Jun 5]; 11(1):80-87. Available from: http://dx.doi.org/10.9789/21755361.rpcfo.vll.6476\%20\%EF\%BB\%BF\%EF\%BB\%BF.

11. Moura RCM, Pereira TF, Rebouças FJ, Costa CM, Lernades AMC, Silva LAK et al. Nursing care in the prevention of obstetric violence. Enferm. Foco [Internet]. 2018 [cited 2020 Abr 09 ]; 9(4):60-65. Available from: http://revista.cofen. gov.br/index.php/enfermagem/article/view/1333/480.

12. Motta SAMF, Feitosa DS, Bezerra STF, Dodt RCM, Moura DJM. Implementation of humanized care to natural childbirth. J Nurs UFPE on line. [Internet]. 2016 [cited 2019 Jun 5]; 2(10):593-599. Available from: http://dx.doi.org/ 10.5205/ reuol.8557-74661-1-SM1002201628.

13. Vargens OMC, Silva ACV, Progianti JM. The contribution of nurse midwives to consolidating humanized childbirth in maternity hospitals in Rio de Janeiro-Brazil. Esc. Anna Nery Rev. Enferm. [Internet]. 2017 [cited 2019 Jun 6]; 21(1):1-8. Available from: http://dx.doi.org/10.5935/1414-8145.20170015. 
14. Barros FRB, Accioly LM, Freitas WFM, Andrade LL, Silva BKC, Araújo RO. Percepção das puérperas manauaras frente à assistência de enfermagem no preparo do trabalho de parto e nascimento. Enferm. Foco [Internet]. 2018 [cited 2020 May 31]; 9(1):76-81. Available from: http://revista.cofen.gov.br/index.php/enfermagem/article/view/1035/432.

15. Andrade LFB, Rodrigues QP, Silva RCV. Good Partices in obstetric care and its interface with humanization of assistance. Revista Enfermagem UERJ [Internet]. 2017 [cited 2019 Jun 6]; 25:e26442. Available from: https://doi.org/10.12957/ reuerj.2017.26442.

16. Monteiro MCM, Holanda VR, Melo GP. Análise do conceito parto humanizado de acordo com o método evolucionário de Rodgers. Revista de Enfermagem do Centro-Oeste Mineiro [Internet]. 2017 [cited 2019 Jun 9 ]; 7:el885. Available from: 10.19175/recom.v7i0.1685.

17. Mselle LT, Kohi TW, Dol J. Barriers and facilitators to humanizing birth care in Tanzania: findings from semi-structured interviews with midwives and obstetricians. Reprod Health [Internet]. 2018 [cited 2020 Abr 9]; 15(1):137. Available from: https://www.ncbi.nlm.nih.gov/pmc/articles/PMC6092851/.

18. World Health Organization (WHO). WHO recommendations: intrapartum care for a positive childbirth experience. [Internet]. Genebra: WHO; 2018. [Internet]. 2018 [cited 2020 Abr 9]. Available from: https://www.who.int/reproductivehealth/publications/intrapartum-care-guidelines/en/.

19. Alencar AJC, Silva AA, Gonçalves MM, Silva DMR, Silva MCT. Nursing care during humanized natural childbirth. Id on Line [Internet]. 2019 [cited 2019 Mai 23]; 13(47):376-38. Available from: http://dx.doi.org/10.14295/idonline.v13i47.2025.

20. Silva FM, Silva ML, Araújo FNP. Sentimentos Causados pela Violência Obstétrica em Mulheres de Municipio do Nordeste Brasileiro. Rev Pre Infec e Saúde [Internet]. 2017 [cited 2019 Jun 9]; 3(4):25-34. Available from: https://doi. org/10.26694/repis.v3i4.6924.

21. Pereira SS, Oliveira ICMS, Santos JBS, Carvalho MCMP. Parto natural: a atuação do enfermeiro diante da assistência humanizada. Tempus actas de saúde colet.[Internet]. 2016 [cited 2019 Jun 9 ]; 10(3):199-213. Available from: http://www. tempusactas.unb.br/index.php/tempus/article/view/1727/1682.

22. Alves TCM, Coelho ASF, Sousa MC, Cesar NF, Silva PS, Pacheco LR. Contributions of the obstetrical nursing for the good practices in labor and vaginal delivery. Enferm. Foco [Internet]. 2019 [cited 2020 Abr 09]; 10(4):54-60. Available from: https://doi.org/10.21675/2357-707X.2019.v10.n4.2210.

23. Ministério da Saúde (BR). Lei n. 11.108, de 7 de abril de 2005. Altera a Lei no 8.080, de 19 de setembro de 1990 , para garantir às parturientes o direito à presença de acompanhante durante o trabalho de parto, parto e pós-parto imediato, no âmbito do Sistema Único de Saúde - SUS. Diário Oficial da República Federativa do Brasil, 07 abr. 2005; 67, seção 1, p. 1. [Internet]. 2005 [cited 2020 Abr 09]. Available from: https://www2.camara.leg.br/legin/fed/lei/2005/lei-11108-7abril-2005-536370 publicacaooriginal-26874-pl.html.

24. Camacho KG, Progianti JM. A transformação da prática obstétrica das enfermeiras na assistência ao parto humanizado. Rev. Eletr. Enf. [Internet]. 2013 [cited 2019 Jun 5]; 15(3):648-55. Available from: http://dx.doi.org/10.5216/ree. v15i3.18588.

25. Dodou HD, Rodrigues DP, Guerreiro EM, Guedes MVC, Lago PN, Mesquita NSA. The contribution of the companion to the humanization of delivery and birth: perceptions of puerperal women. Esc. Anna Nery Rev. Enferm. [Internet]. 2014 [cited 2019 Jun 9]; 18(2):282-269. Available from: http://dx.doi.org/10.5935/1414-8145.20140038.

26. Giraldo D, González E, Henao C. Experiencias de las mujeres durante el trabajo de parto y parto. Av. Enferm. [Internet] 2015 [cited $2020 \mathrm{Abr}$ 9]; 33(2):271-81. Available from: https://revistas.unal.edu.co/index.php/avenferm/article/ view/42279/54803. 